\title{
Epigenome of Malus domestica
}

\author{
Andrea GULYÁS ${ }^{1}$ - Judit DOBRÁNSZKI ${ }^{1}$ - Erzsébet $\mathrm{KISS}^{2}$ - Katalin POSTA ${ }^{2}$ - \\ Norbert HIDVÉGI ${ }^{1}$ \\ 1: Research Institute of Nyíregyháza, IAREF, University of Debrecen, Nyíregyháza, P.O. Box 12, H-4400, \\ Hungary; gulyas.andrea@agr.unideb.hu \\ 2: Institute of Genetics, Microbiology and Biotechnology, Faculty of Agricultural and Environmental Sciences, \\ Szent István University, Gödöllő, Páter Károly u.1., H-2100, Hungary; kiss.erzsebet@mkk.szie.hu
}

Keywords: apple, epigenetic, DNA methylation, gene expression

\section{Introduction}

The aim of this study was to examine the epigenomes of two diploid apple scion cultivars from three distinct environments: 1) in vitro shoots maintained for 16 years in tissue culture; 2 ) in vivo mother trees (20 years old); 3) acclimatized in vitro plants (one year after acclimatization). Using whole-genome bisulfite sequencing (WGBS), the level of DNA methylation was measured in these three biological samples to determine whether an epigenetic footprint was left within the epigenome of apple due to different environments (in vivo mother tree vs. in vitro) or a change in the environment (in vitro culture to acclimatized stage). The study further assessed if the DNA methylation pattern of acclimatized plants mirrored that of the original parental material (i.e., in vivo mother plant vs. one year after acclimatization).

\section{Materials and methods}

Plant material was collected from three sources. The first source was in vitro leaves from four-week-old in vitro shoot cultures of Malus $x$ domestica Borkh. scion cultivars 'McIntosh' and 'Húsvéti rozmaring'. Shoot cultures were maintained for 16 years and subcultured monthly on basal medium (Magyar-Tábori et al., 2009). The second source was in vivo leaves collected from in vivo mother trees of both cultivars from which in vitro cultures had been originally established 16 years earlier. The third source was leaves collected from rooted in vitro apple shoots one year after acclimatization (Magyar-Tábori et al., 2009). DNA was extracted and purified from all three samples of each cultivar with a NucleoSpin plant II DNA extraction kit (Macherey-Nagel, Düren, Germany), following the manufacturer's instructions. Bisulfite treatment was applied with the Pico MethylSeq Library Prep kit (Zymo Research, Irvine, CA, USA) based on the user manual. WGBS was performed on a Illumina HiSeq 2500 (Illumina, San Diego, CA, USA). Differential methylation, statistical analysis, DNA methylation distribution plots and gene clustering were performed with SeqMonk v1.41.0 (https://github.com/s-andrews/SeqMonk). Genes that displayed significant differences in DNA methylation in either their promoter or coding regions according to the $\chi 2$ test were classified as differentially methylated genes (DMGs). All assembled DMGs (based on the $\chi 2$ test) were considered for functional mapping of biological processes, molecular functions and cellular compounds. The functions of all DMGs in the three environments were determined, focusing on biological processes, molecular functions and cellular components of green plants (Viridiplantae), as these might have important roles during in vitro culture by Gene Ontology (GO) annotation with Blast2GO v5.1.12 (Conesa and Götz, 2008). 


\section{Results and discussion}

In the present study on apple, when studying the level of global DNA methylation, no significant differences were found in the degree of methylated cytosine positions between apple scion cultivars. Analysis of DNA methylation at the level of the entire genome showed significant differences in $\mathrm{C}$ methylation between some genes in either their promoters or coding regions. A total of 45,116 genes, including their promoters and coding regions, were studied. Significant differences in DNA methylation were identified in 586 genes, i.e. DMGs, specifically 334, 201, and 131 in $\mathrm{CpG}$, CHG and CHH contexts. The level of methylation in DMGs decreased after acclimatization. In vitro tissue culture had the highest level of methylated DMGs. Some DMGs that participate in oxidation-reduction processes, metabolism and biosynthesis. Methylation patterns of the two scion cultivars differed, indicating cultivar-specific regulation of the epigenome during the adaptation of apple to various environments. The level of DNA methylation of DMGs was lower in 'McIntosh' than in 'Húsvéti rozmaring', which might indicate differences in adapting to an in vitro environment. According to GO annotation of all DMGs, a total of 235, 310 and 189 DMGs play important roles in biological processes, molecular functions and cellular components, respectively.

\section{Conclusions}

Our experiments show that the levels of global genomic DNA methylation in apple were steady, independent of the cultivar or growth environment. However, analyses of the methylation pattern in the entire genome confirmed that individual genes display constantly changing levels of methylation. The dynamic changes in their methylation levels might regulate responses and adaptation to a changing environment (in vitro environment or recovery to the in vivo environment). After acclimatization, the pattern of DNA methylation in the two apple cultivars were similar to the methylation pattern of the mother plant.

\section{Acknowledgement}

The research was financed by the Higher Education Institutional Excellence Programme of the Ministry of Human Capacities in Hungary, within the framework of the Biotechnology thematic programme of the University of Debrecen (20428-3/2018/FEKUTSTRAT), and that of Szent Istvan University (1783-3/2018/FEKUTSTART).

\section{References}

Conesa, A. \& Götz, S. (2008): Blast2GO: A comprehensive suite for functional analysis in plant genomics. International Journal of Plant Genomics 2008, 619832 . https://doi.org/10.1155/2008/619832

Magyar-Tábori, K., Dobránszki, J., Hudák, I. (2009): Factors affecting the rooting ability of in vitro apple shoots. Hungarian Agricultural Research 18.10, 4. https://doi.org/10.1016/j.scienta.2011.05.011 